\title{
THE NONCOMMUTATIVITY OF HECKE ALGEBRAS ASSOCIATED TO WEYL GROUPS
}

\author{
MICHAEL R. ANDERSON
}

(Communicated by Lance W. Small)

\begin{abstract}
We prove that the Hecke algebra $\mathscr{H}\left(W, W_{J}\right)$, where $W$ is a Weyl group of spherical type and $W_{J}$ is a standard parabolic subgroup of $W$ of corank $\geq 2$, is noncommutative.
\end{abstract}

\section{INTRODUCTION}

Let $G$ be a finite group, $H$ a subgroup of $G$, and $K$ a field of characteristic zero. Let $\mathscr{H}(G, H)$ be the Hecke algebra of $G$ with respect to $H$, defined over $K$. It may be viewed as the algebra of double cosets of $H$ in $G$ (see [K] for details) or, equivalently, as the subalgebra $e_{H} K[G] e_{H}$ of $K[G]$, where $e_{H}=(1 / \# H) \sum_{h \in H} h$.

A natural question to ask about $\mathscr{H}$ is whether it is commutative. When $G$ is a Weyl group, Iwahori gave the following theorem that tells when $\mathscr{H}$ is commutative:

Theorem 1.1 ([I, Theorem 2]). Let $W$ be a Weyl group and let $W_{J}$ be a parabolic subgroup of $W . \mathscr{H}\left(W, W_{J}\right)$ is commutative if and only if $W_{J} w W_{J}=$ $W_{J} w^{-1} W_{J}$ for all $w \in W$.

Let $W$ be a Weyl group of spherical type. It has been known for some time now which maximal parabolic subgroups $W_{J}$ of $W$ give rise to commutative Hecke algebras (see, for example, Theorem 10.4.11 of [BCN]). In this paper, we examine the case where $W_{J}$ is a nonmaximal parabolic subgroup of $W$ and prove the following:

Theorem 1.2. Let $(W, R)$ be a Weyl group of type $B_{n}, D_{n}, E_{6}, E_{7}, E_{8}, F_{4}$ or $G_{2}$ with generating set $R=\left\{r_{1}, r_{2}, \ldots, r_{n}\right\}$. Let $J \subset R$. If $\#(R \backslash J) \geq 2$, then $\mathscr{H}\left(W, W_{J}\right)$ is noncommutative.

That $\mathscr{H}\left(W, W_{J}\right)$, where $W$ is of type $A_{n}$ and $W_{J}$ is a nonmaximal parabolic, is noncommutative is already known (see Lemma III.3.5 of [K]), so we will not consider it.

Write the Coxeter diagrams as on [Su, p. 306] and number the generators from left to right.

Received by the editors August 10, 1993 and, in revised form, December 12, 1993.

1991 Mathematics Subject Classification. Primary 16S99; Secondary 20 C33. 
If $W$ is a Weyl group with generators $R=\left\{r_{1}, r_{2}, \ldots, r_{n}\right\}$, and $J \subset R$, we say $W_{J}$ has corank $k$ if $\#\{R \backslash J)=k$. We denote by $P_{i}$ the parabolic subgroup of $W$ generated by $R \backslash\left\{r_{i}\right\}$, by $P_{i j}$ the parabolic subgroup of $W$ generated by $R \backslash\left\{r_{i}, r_{j}\right\}$, etc.

We begin by introducing data about $\mathscr{H}\left(W, W_{J}\right)$ for various $W$ and $W_{J}$, derived using CAYLEY software. From this data, and Theorems 2.1 and 3.2, we construct the proof of Theorem 1.2. In the following tables, Dimension refers to the dimension of the given Hecke algebra, while Dimension of invariants refers to the dimension of the set of elements of $\mathscr{H}\left(W, W_{J}\right)$ which are fixed by the canonical involution. The column $w$ gives an element of $W$ for which $W_{J} w W_{J} \neq W_{J} w^{-1} W_{J}$.

What Theorem 1.1 means for us is that if the numbers in the Dimension and Dimension of invariants columns are different, then $\mathscr{H}\left(W, W_{J}\right)$ is noncommutative.

\section{THE EXCEPTIONAL WeYl GROUPS}

Table 1 lists data for selected Hecke algebras of corank 2 subgroups in $F_{4}$, $E_{6}, E_{7}$ and $E_{8}$, computed using CAYLEY. Those cases not listed in Table 1 are noncommutative, too. $\mathscr{H}\left(G_{2}, P_{12}\right)$ is noncommutative because $P_{12}=\langle e\rangle$, so $\mathscr{H}\left(G_{2}, P_{12}\right)=K\left[G_{2}\right]$. The others arise from subgroups that are contained in subgroups that give rise to noncommutative Hecke algebras (see Theorem 10.4.11 of $[\mathrm{BCN}]$ or Table 1 of $[\mathrm{A}]$ ), so they also are noncommutative by the following theorem, which also implies that all Hecke algebras of subgroups in $F_{4}, E_{6}, E_{7}$ and $E_{8}$ of corank $\geq 3$ are noncommutative.

Theorem 2.1. Let $G$ be a finite group. Let $S, T$ be subgroups of $G$ with $T \subset S$. If $\mathscr{H}(G, S)$ is noncommutative, then $\mathscr{H}(G, T)$ is noncommutaiive.

Proof. Let us view $\mathscr{H}(G, S)$ and $\mathscr{H}(G, T)$ as $e_{S} K[G] e_{S}$ and $e_{T} K[G] e_{T}$. Let $\left\{T_{i}\right\}$ be the standard basis of $e_{T} K[G] e_{T}$. By Lemma 2.3 of [CIK], we can form a basis $\left\{Z_{j}\right\}$ of $e_{S} K[G] e_{S}$ by summing elements of $\left\{T_{i}\right\}$, i.e.,

$$
Z_{j}=\sum_{i \in J_{j}} T_{i},
$$

for some index set $J_{j}$.

Suppose $\mathscr{H}(G, T)\left(\cong e_{T} K[G] e_{T}\right)$ is commutative. Then for all $i_{1}$ and $i_{2}$, $T_{i_{1}} \cdot T_{i_{2}}=T_{i_{2}} \cdot T_{i_{1}}$. Hence, for any $j_{1}$ and $j_{2}$,

$$
\begin{aligned}
Z_{j_{1}} \cdot Z_{j_{2}} & =\left(\sum_{i \in J_{j_{1}}} T_{i}\right)\left(\sum_{i \in J_{j_{2}}} T_{i}\right)=\sum_{\substack{i \in J_{j_{1}} \\
j \in J_{j_{2}}}} T_{i} \cdot T_{j} \\
& =\sum_{\substack{i \in J_{j_{1}} \\
j \in J_{j_{2}}}} T_{j} \cdot T_{i}=\left(\sum_{i \in J_{j_{2}}} T_{i}\right)\left(\sum_{i \in J_{j_{1}}} T_{i}\right)=Z_{j_{2}} \cdot Z_{j_{1}} .
\end{aligned}
$$

Thus, $\mathscr{H}(G, S)\left(\cong e_{S} K[G] e_{S}\right)$ is commutative. 
TABLE 1. Hecke algebras relative to corank 2 subgroups of $F_{4}, E_{6}, E_{7}, E_{8}$.

\begin{tabular}{cccc}
$\begin{array}{c}\text { Hecke } \\
\text { algebra }\end{array}$ & Dimension & $\begin{array}{c}\text { Dimension } \\
\text { of } \\
\text { invariants }\end{array}$ & $w$ \\
\hline $\mathscr{H}\left(F_{4}, P_{14}\right)$ & 33 & 25 & $r_{1} r_{2} r_{3} r_{4}$ \\
$\mathscr{H}\left(E_{6}, P_{14}\right)$ & 26 & 20 & $r_{1} r_{2} r_{3} r_{4}$ \\
$\mathscr{H}\left(E_{6}, P_{16}\right)$ & 21 & 16 & $r_{1} r_{2} r_{3} r_{5} r_{6}$ \\
$\mathscr{H}\left(E_{6}, P_{46}\right)$ & 26 & 20 & $r_{4} r_{3} r_{5} r_{6} r_{4}$ \\
$\mathscr{H}\left(E_{7}, P_{15}\right)$ & 70 & 52 & $r_{1} r_{2} r_{3} r_{4} r_{5}$ \\
$\mathscr{H}\left(E_{7}, P_{17}\right)$ & 36 & 28 & $r_{1} r_{2} r_{3} r_{4} r_{6} r_{7}$ \\
$\mathscr{H}\left(E_{7}, P_{57}\right)$ & 70 & 52 & $r_{5} r_{4} r_{6} r_{7} r_{5}$ \\
$\mathscr{H}\left(E_{8}, P_{18}\right)$ & 114 & 80 & $r_{1} r_{2} r_{3} r_{4} r_{5} r_{7} r_{8}$
\end{tabular}

Note. One may also use Theorem 3.2 to show the noncommutativity of all of the Hecke algebras listed in Table 1, except for $\mathscr{H}\left(F_{4}, P_{14}\right)$.

\section{3. $D_{n}$}

The next few results show that for all $n$, and for all $i, j$ with $1 \leq i<j \leq n$, $\mathscr{H}\left(D_{n}, P_{i j}\right)$ is noncommutative.

Proposition 3.1. Let $(W, R)$ be a Coxeter system of finite rank. Let $I, J \subset R$, and let $w \in W_{J}$. Then $W_{I} w W_{I} \cap W_{J}=\left(W_{I} \cap W_{J}\right) w\left(W_{I} \cap W_{J}\right)$.

Proof. Analogous to the proof of Corollary 12.8 of [T].

Theorem 3.2. Let $(W, R)$ and $\left(W^{\prime}, R^{\prime}\right)$ be Weyl groups such that $R^{\prime} \subseteq R$. Let $J^{\prime} \subseteq R^{\prime}$. Let $J=J^{\prime} \cup\left(R \backslash R^{\prime}\right)$. If $\mathscr{H}\left(W^{\prime}, W_{J^{\prime}}^{\prime}\right)$ is noncommutative, then so is $\mathscr{H}\left(W, W_{J}\right)$.

Proof. Suppose $\mathscr{H}\left(W, W_{J}\right)$ is commutative. Then by Theorem 1.1, $W_{J} w W_{J}$ $=W_{J} w^{-1} W_{J}$ for all $w \in W$.

Let $w \in W^{\prime}$. By Proposition 3.1,

$$
\begin{aligned}
W_{J^{\prime}}^{\prime} w W_{J^{\prime}}^{\prime} & =W_{J} w W_{J} \cap W^{\prime} \\
& =W_{J} w^{-1} W_{J} \cap W^{\prime} \\
& =W_{J^{\prime}}^{\prime} w^{-1} W_{J^{\prime}}^{\prime} .
\end{aligned}
$$

Hence, by Theorem 1.1, $\mathscr{H}\left(W^{\prime}, W_{J^{\prime}}^{\prime}\right)$ is commutative.

We now continue our analysis of $D_{n}$.

Let $n \geq 4$. We wish to show that $\mathscr{H}\left(D_{n}, P_{i j}\right)$ is noncommutative for all $1 \leq i<j \leq n$. Let $i, j \in\{1,2, \ldots, n\}, i \neq j$. At least one of $\left\{r_{1}, r_{n-1}, r_{n}\right\}$ is in $R \backslash\left\{r_{i}, r_{j}\right\}$. We have two cases to consider: 
Case 1. $r_{n-1}$ or $r_{n}$ in $R \backslash\left\{r_{i}, r_{j}\right\}$.

Let $W^{\prime}$ be the group generated by $R^{\prime}$, where $R^{\prime}=R \backslash\left\{r_{n-1}\right\}$ or $R^{\prime}=$ $R \backslash\left\{r_{n}\right\} . W^{\prime}$ is isomorphic to $A_{n-1}$.

Let $P_{i j}^{\prime}$ be the parabolic subgroup of $W^{\prime}$ generated by. $R^{\prime} \backslash\left\{r_{i}, r_{j}\right\}$. By Lemma III.3.5 of $[\mathrm{K}], \mathscr{H}\left(W^{\prime}, P_{i j}^{\prime}\right)$ is noncommutative. Hence, by Theorem 3.2, $\mathscr{H}\left(D_{n}, P_{i j}\right)$ is noncommutative.

Case 2. $i=n-1, j=n$.

We will induct on $n$, beginning with $n=4$. For $n=4$, let $W^{\prime}=$ $\left\langle r_{2}, r_{3}, r_{4}\right\rangle$. $W^{\prime}$ is isomorphic to $A_{3}$. Let $P^{\prime}=\left\langle r_{2}\right\rangle$. By Lemma III.3.5 of $[\mathrm{K}], \mathscr{H}\left(W^{\prime}, P^{\prime}\right)$ is noncommutative, so by Theorem $3.2, \mathscr{H}\left(D_{4}, P_{34}\right)$ is noncommutative.

Suppose that $\mathscr{H}\left(D_{k}, P_{k-1, k}\right)$ is noncommutative for all $4 \leq k<n$. Let $W^{\prime}$ be generated by $R^{\prime}=\left\{r_{2}, r_{3}, \ldots, r_{n}\right\}$, and $P^{\prime}$ be generated by $R^{\prime} \backslash\left\{r_{n-1}, r_{n}\right\}$. $W^{\prime}$ is isomorphic to $D_{n-1}$, so by induction, $\mathscr{H}\left(W^{\prime}, P^{\prime}\right)$ is noncommutative. Hence, by Theorem $3.2, \mathscr{H}\left(D_{n}, P_{n-1, n}\right)$ is noncommutative.

\section{4. $B_{n}$}

We now turn to $B_{n}$. Table 2 contains data about Hecke algebras with respect to corank 2 subgroups of $B_{3}$ and $B_{4}$.

As before, we see that these corank 2 parabolics give rise to noncommutative Hecke algebras. We also know that $\mathscr{H}\left(B_{2}, P_{12}\right)$ is noncommutative, since $P_{12}=\langle e\rangle$, so

$$
\mathscr{H}\left(B_{2}, P_{12}\right) \cong K\left[B_{2}\right],
$$

which is noncommutative. We will now prove that $\mathscr{H}\left(B_{n}, P_{i j}\right)$ is noncommutative for all $n \geq 2$ and all parabolic subgroups of the form $P_{i j}$, where $i<j$.

TABLE 2. Hecke algebras relative to corank 2 subgroups of $B_{3}, B_{4}$.

\begin{tabular}{cccc}
$\begin{array}{c}\text { Hecke } \\
\text { algebra }\end{array}$ & Dimension & $\begin{array}{c}\text { Dimension } \\
\text { of } \\
\text { invariants }\end{array}$ & $w$ \\
\hline $\mathscr{H}\left(B_{3}, P_{12}\right)$ & 16 & 13 & $r_{1} r_{2}$ \\
$\mathscr{H}\left(B_{3}, P_{13}\right)$ & 14 & 12 & $r_{1} r_{2} r_{3}$ \\
$\mathscr{H}\left(B_{3}, P_{23}\right)$ & 14 & 12 & $r_{2} r_{3}$ \\
$\mathscr{H}\left(B_{4}, P_{12}\right)$ & 17 & 14 & $r_{1} r_{2}$ \\
$\mathscr{H}\left(B_{4}, P_{13}\right)$ & 33 & 26 & $r_{1} r_{2} r_{3}$ \\
$\mathscr{H}\left(B_{4}, P_{14}\right)$ & 20 & 17 & $r_{1} r_{2} r_{3} r_{4}$ \\
$\mathscr{H}\left(B_{4}, P_{23}\right)$ & 33 & 26 & $r_{2} r_{3}$ \\
$\mathscr{H}\left(B_{4}, P_{24}\right)$ & 34 & 27 & $r_{2} r_{3} r_{4}$ \\
$\mathscr{H}\left(B_{4}, P_{34}\right)$ & 20 & 17 & $r_{3} r_{4}$
\end{tabular}


Let $W^{\prime}$ be generated by $R^{\prime}=R \backslash\left\{r_{n}\right\} . W^{\prime}$ is isomorphic to $A_{n-1}$. Let $P_{i j}^{\prime}$ be generated by $R^{\prime} \backslash\left\{r_{i}, r_{j}\right\}$. By Lemma III.3.5 of $[\mathrm{K}], \mathscr{H}\left(W^{\prime}, P_{i j}^{\prime}\right)$ is noncommutative, so by Theorem $3.2, \mathscr{H}\left(B_{n}, P_{i j}\right)$ is noncommutative.

The remaining case to consider is $\mathscr{H}\left(B_{n}, P_{i n}\right), i<n$. Suppose that $i>1$. If we let $W^{\prime}$ be generated by $R^{\prime}=R \backslash\left\{r_{1}, r_{2}, \ldots, r_{i-1}\right\}$, then by Theorem 3.2 , we would just need to show that $\mathscr{H}\left(B_{n}, P_{1 n}\right)$ is noncommutative for all $n \geq 2$. However, if we try to use Theorem 3.2 to do this, we run into the following problem: Let $n>4$. Let $1<k<n$. Let $W^{\prime}$ be generated by $R^{\prime}=R \backslash\left\{r_{k}\right\}$. Then $W^{\prime}$ is isomorphic either to $A_{k-1} \times B_{n-k}$ or to $A_{n-2} \times A_{1}$, depending on $k$. Let $P^{\prime}$ be generated by $R^{\prime} \backslash\left\{r_{1}, r_{n}\right\}$, so $P^{\prime}$ is isomorphic either to $A_{k-2} \times A_{n-k-1}$ or to $A_{n-3} \times\langle e\rangle$, respectively. By Theorem I.6.3 of [K], $\mathscr{H}\left(W^{\prime}, P^{\prime}\right)$ is isomorphic either to $\mathscr{H}\left(A_{k-1}, A_{k-2}\right) \otimes \mathscr{H}\left(B_{n-k}, A_{n-k-1}\right)$ or to $\mathscr{H}\left(A_{n-2}, A_{n-3}\right) \otimes \mathscr{H}\left(A_{1},\langle e\rangle\right)$. In either case, by Proposition III.3.3 of $[\mathrm{K}]$ and [CIK], both factors of the tensor product are commutative, so $\mathscr{H}\left(W^{\prime}, P^{\prime}\right)$ is commutative. But Theorem 3.2 can be used only if $\mathscr{H}\left(W^{\prime}, P^{\prime}\right)$ is noncommutative. Thus, we need another approach. A closer look at the root system for $B_{n}$ will provide the needed tools.

Proposition 4.1. Let $n \geq 2$. $\mathscr{H}\left(B_{n}, P_{1 n}\right)$ is noncommutative.

Proof. Let $\left\{\varepsilon_{i}\right\}_{i=1}^{n}$ be the standard basis of $\mathbb{R}^{n}$. Let $\alpha_{i}=\varepsilon_{i}-\varepsilon_{i+1}$ for $1 \leq$ $i \leq n-1$ and let $\alpha_{n}=\varepsilon_{n}$. By Section 12.1 of [Hu], $\left\{\alpha_{i}\right\}_{i=1}^{n}$ forms a base of the root system of type $B_{n}$. By Theorem 10.3 of [Hu], the standard reflections $\sigma_{i}=\sigma_{\alpha_{i}}, i=1,2, \ldots, n$, generate the Weyl group $W$ of type $B_{n}$, where for $1 \leq i \leq n-1, \sigma_{i}$ acts by permuting $\varepsilon_{i}$ and $\varepsilon_{i+1}$ while $\sigma_{n}\left(\varepsilon_{n}\right)=-\varepsilon_{n}$. Thus, everything in $W$ permutes and/or changes the signs of the $\varepsilon_{i}$, for $i=$ $1,2, \ldots, n$. Let $w=\sigma_{1} \sigma_{2} \cdots \sigma_{n}$ (the Coxeter element). By an easy calculation (working right-to-left), $w\left(\varepsilon_{1}\right)=\varepsilon_{2}$, while $w^{-1}\left(\varepsilon_{1}\right)=-\varepsilon_{n}$.

Let $P_{1 n}$ be the parabolic subgroup of $W$ generated by $\left\{\sigma_{2}, \ldots, \sigma_{n-1}\right\}$. Let $w_{1}, w_{2} \in P_{1 n}$. Since $w_{2} \in P_{1 n}$, it is the product of elements of $\left\{\sigma_{2}, \ldots, \sigma_{n-1}\right\}$, so it fixes $\varepsilon_{1}$, i.e., $w_{2}\left(\varepsilon_{1}\right)=\varepsilon_{1}$. Hence, $w w_{2}\left(\varepsilon_{1}\right)=w\left(\varepsilon_{1}\right)=\varepsilon_{2}$.

Since $w_{1} \in P_{1 n}, \sigma_{n}$ does not appear in its decomposition, so $w_{1}\left(\varepsilon_{i}\right)=$ $\varepsilon_{j}, \forall i=1,2, \ldots, n$ and some $j \in\{1,2, \ldots, n\}$. The important point here is that $\varepsilon_{j}$ is a positive linear combination of the fundamental roots. In particular, $w_{1}\left(\varepsilon_{2}\right)$ will be a positive linear combination of fundamental roots, so $w_{1} w w_{2}\left(\varepsilon_{1}\right)=w_{1}\left(\varepsilon_{2}\right)$ is also one. On the other hand, we know $w^{-1}\left(\varepsilon_{1}\right)=-\varepsilon_{n}$, i.e., $w^{-1}\left(\varepsilon_{1}\right)$ is not one. Therefore,

$$
w_{1} w w_{2} \neq w^{-1} \quad \forall w_{1}, w_{2} \in P_{1 n} .
$$

Hence, $P_{1 n} w P_{1 n} \neq P_{1 n} w^{-1} P_{1 n}$, so by Theorem $1.1, \mathscr{H}\left(B_{n}, P_{1 n}\right)$ is noncommutative.

As for the parabolic subgroups of corank $>2$, since they all occur as subgroups of parabolics of corank 2, Theorem 2.1 will imply that they also all give rise to noncommutative Hecke algebras.

Note. These results have some interest for the representation theory of Weyl groups. As is well known, $\mathscr{H}\left(W, W_{J}\right)$ is the centralizer algebra of the induced representation $1_{W_{J}}^{W}$, so the commutativity of $\mathscr{H}$ tells whether this representation is multiplicity-free. Hence, one way to express our results is to say that for any classical Weyl group $W$ and parabolic subgroup $P$ of corank $\geq 2$, 
$1_{P}^{W}$ is not multiplicity-free. This is also the point of interest for the theory of distance-regular graphs.

Note. The data used in compiling Tables 1 and 2 are available from the author. For a discussion of the algorithms used, see [A].

\section{ACKNOWLEDGMENTS}

This paper is based on Chapter V of [A]. I would like to take this opportunity to thank Professor Joseph C. Ferrar of Ohio State University for serving as my advisor. I would also like to thank the referee for bringing the reference [I] to my attention and for his suggestions for improving the paper.

\section{REFERENCES}

[A] M. R. Anderson, Hecke algebras associated to Weyl groups, Ph.D. thesis, Ohio State University, 1993.

[BC] W. Bosma and J. Cannon, Handbook of Cayley functions, Sydney, 1992.

[BCN] A. E. Brouwer, A. M. Cohen, and A. Neumaier, Distance-regular graphs, Springer-Verlag, Berlin and New York, 1989.

[CIK] C. W. Curtis, N. Iwahori, and R. Kilmoyer, Hecke algebras and characters of parabolic type of finite groups with BN-pairs, Inst. Hautes Études Sci. Publ. Math. 40 (1971), 81-116.

[Hu] J. E. Humphreys, Introduction to Lie algebras and representation theory, Springer-Verlag, Berlin and New York, 1972.

[I] N. Iwahori, On some properties of groups with BN-pairs, Theory of Finite Groups (Brauer and Sah, eds.), Benjamin, New York, 1969.

[K] A. Krieg, Hecke algebras, Mem. Amer. Math. Soc. 87 (1990), No. 435.

[Su] M. Suzuki, Group theory. I, Springer-Verlag, Berlin and New York, 1982.

[T] J. Tits, Buildings of spherical type and finite BN-pairs, Lecture Notes in Math., vol. 386, Springer-Verlag, Berlin and New York, 1974.

Department of Mathematics, Ohio State University, Columbus, Ohio 43210

E-mail address: anderson@math.ohio-state.edu 